\title{
Mucormycosis causing massive lower gastrointestinal bleeding: a case report
}

\author{
Ting-Hsuan Chiang ${ }^{1}$, Yi-Wei Lee ${ }^{1}$, Jui-Hsiang Tan², Chih-Chin Kao ${ }^{2,3}$, Chun-Chao Chang ${ }^{2,3}$ and \\ Kuan-Chieh Fang ${ }^{2,3^{*}}$ (D)
}

\begin{abstract}
Background: Lower gastrointestinal bleeding $(L G \mid B)$ is very common in the hospital setting. Most bleedings stop spontaneously, but rare infectious causes of LGIB may lead to rapid and serious complications if left untreated and are sometimes very difficult to diagnose preoperatively.

Case presentation: We described a young man with poorly controlled Type I diabetes mellitus and chronic alcohol abuse who presented with acute altered mental status. During his hospitalization for treatment of diabetic ketoacidosis, acute renal failure, and sepsis, he suddenly developed massive hematochezia of $1500 \mathrm{~mL}$. Colonoscopy was performed and a deep ulcer covered with mucus with peripheral elevation was noted at the transverse colon. Biopsy of the ulcer later revealed nonpigmented, wide (5-20 $\mu \mathrm{m}$ in diameter), thin-walled, ribbon-like hyphae with few septations and right-angle branching suggestive of mucormycosis demonstrated by Periodic acid-Schiff stain. He received 2 months of antifungal treatment. Follow up colonoscopy post-treatment was normal with no ulcer visualized.

Conclusions: Early diagnosis and treatment of gastrointestinal (GI) mucormycosis infection is critical but can be challenging, especially in the setting of massive hematochezia. Therefore, clinical awareness for immunocompromised patients and prompt antifungal prophylaxis in cases with high suspicion of infection are essential.
\end{abstract}

Keywords: Mucormycosis, Hematochezia, Diabetic ketoacidosis, Opportunistic infection, Case report

\section{Background}

Lower gastrointestinal bleeding (LGIB) presenting as hematochezia is very common in the hospital setting. Most cases of LGIB stop spontaneously, but acute severe bleeding can be life-threatening, especially in patients with comorbidities. To improve diagnostic and therapeutic yields, early colonoscopy is recommended for patients with acute LGIB [1].

The most common etiologies of LGIB include diverticulosis, angiodysplasia, colorectal neoplasms, hemorrhoids, and rectal ulcer. Other causes such as inflammatory bowel disease and proctitis are also important differential

\footnotetext{
*Correspondence: midhill123@gmail.com

2 Division of Gastroenterology, Internal Medicine, Taipei Medical

University Hospital, No. 252, Wuxing Street, Taipei, Taiwan, ROC

Full list of author information is available at the end of the article
}

diagnoses. In immunocompromised patients, opportunistic infections of the GI tract resulting in massive LGIB have been reported in previous literature. These include viral infections such as cytomegalovirus colitis [2], bacterial infections such as tuberculosis [3], and fungal infections such as aspergillosis [4] and mucormycosis [5]. Among these infections, mucormycosis causing LGIB has been the least reported.

Presentations of intestinal mucormycosis reported in literature have been non-specific, ranging from afebrile abdominal pain to severe intestinal perforation [6]. In the few reported cases of mucormycosis presenting as severe LGIB, almost all patients died despite treatment [5, 7]. Delayed diagnosis was often identified as the fundamental reason for mortality [8]. We described a case of GI mucormycosis presenting as massive hematochezia in an immunocompromised patient with diabetic ketoacidosis 
(DKA). Prompt diagnosis with colonoscopy and antifungal treatment resulted in satisfactory outcome.

\section{Case presentation}

A 28-year-old man was brought to the Emergency Department (ED) by his father due to acute altered mental status and shortness of breath since earlier that morning. His medical history was significant for poorly controlled Type I Diabetes Mellitus with multiple hospitalizations due to diabetes ketoacidosis (DKA), severe chronic alcohol abuse for 8 years, and chronic pancreatitis. He was also diagnosed with nasopharyngeal carcinoma at age 18, for which he underwent concurrent chemoradiotherapy. According to his father, he had not eaten food since the night prior but consumed large amounts of alcohol. Upon arrival at the ED, the patient was stuporous, tachypneic at 26 breaths per minute, blood pressure 134/109 mmHg, heart rate 85 beats per minute, and afebrile. Initial blood work showed glucose of $420 \mathrm{mg} / \mathrm{dL}$, high levels of alcohol $(222.7 \mathrm{mg} / \mathrm{dL})$, metabolic acidosis, elevated ketones $(5.4 \mathrm{mmol} / \mathrm{L})$, pancytopenia (WBC: $1.03 \times 10^{3} / \mathrm{uL}$, Hemoglobin: $9.9 \mathrm{~g} / \mathrm{dL}$, Platelet count: $\left.32 \times 10^{3} / \mathrm{uL}\right)$ prolonged prothrombin time (19.8 s) and activated partial prothrombin time ( $>180 \mathrm{~s})$, hyperammonemia (876 ug/dL), elevated lactate $(19.9 \mathrm{mmol} / \mathrm{L})$, elevated liver enzymes (AST 3112 U/L, ALT $328 \mathrm{U} / \mathrm{L}$, Gamma-glutamyl-transferase $1100 \mathrm{U} / \mathrm{L}$ ), elevated creatinine $(2.8 \mathrm{mg} / \mathrm{dL})$ and Blood urea nitrogen $(22.0 \mathrm{mg} / \mathrm{dL})$. Initial treatment for his DKA state was given at the ED. However, $3 \mathrm{~h}$ later, the patient developed hypotension $(\mathrm{BP}=82 / 42)$. Norepinephrine was given and the patient was subsequently admitted to the Intensive Care Unit (ICU) for further workup and treatment.

At the ICU, the patient was intubated due to hypoxemic respiratory failure. He underwent continuous renal replacement therapy owing to acute kidney injury and severe metabolic acidosis. Empiric antibiotics were prescribed because of suspected sepsis (Sequential Organ Failure Assessment Score: 9). His blood culture further confirmed growth of Pseudomonas Aeruginosa and Acinetobacter Baumannii. Antibiotics were adjusted according to susceptibility. Additional blood work revealed negative HBV, HCV, and HIV antibodies. His condition gradually improved and was successfully extubated on day 10.

However, on day 11 of admission, massive hematochezia of $1500 \mathrm{~mL}$ was noted, and his hemoglobin dropped from 9.5 to $8.2 \mathrm{~g} / \mathrm{dL}$. Blood transfusion, vitamin $\mathrm{K}$, and tranexamic acid were immediately administered. Being hemodynamically stable the next day, the patient underwent colonoscopy. During the procedure, other than blood clots and stool visualized along the proceeding scope, a deep ulcer covered with mucus with peripheral

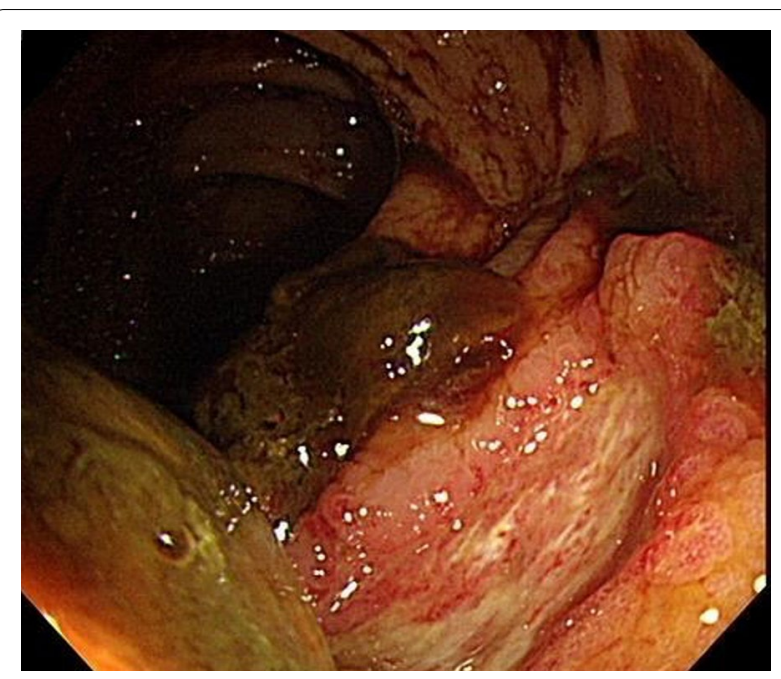

Fig. 1 A deep ulcer covered with mucus with peripheral elevation was noted at the transverse colon

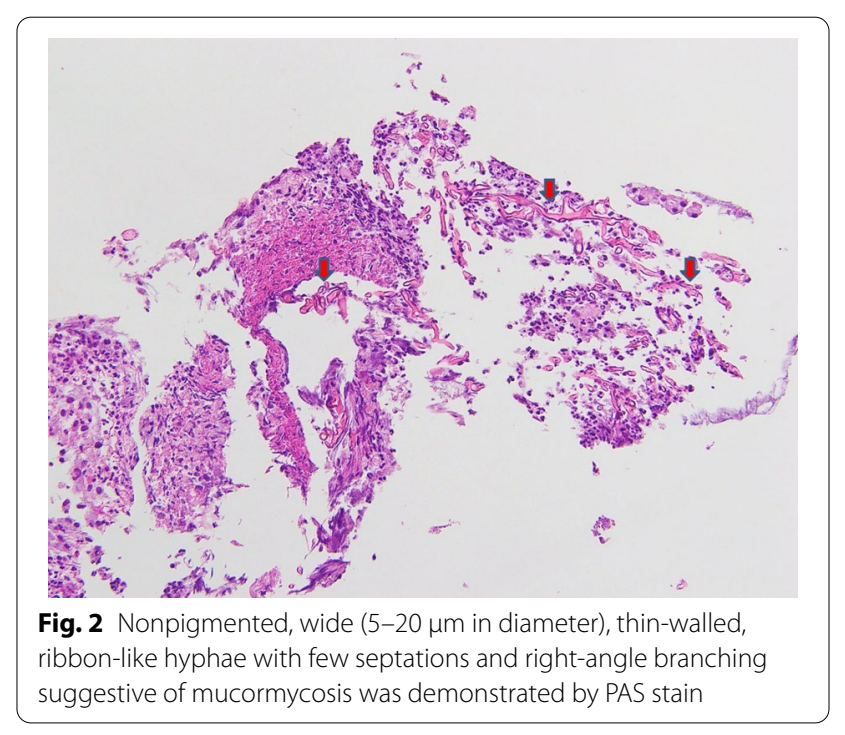

elevation was noted at the transverse colon (Fig. 1). A biopsy was done and the specimen was sent to pathology. Histopathology of the biopsy revealed nonpigmented, wide $(5-20 \mu \mathrm{m}$ in diameter), thin-walled, ribbon-like hyphae with few septations and right-angle branching suggestive of mucormycosis demonstrated by Periodic acid-Schiff (PAS) stain (Fig. 2). Blood cultures taken at that time were negative and upper endoscopy did not reveal similar lesions.

Upon receiving the pathology report 5 days after the colonoscopy, the patient was started on oral Posaconazole therapy. No hematochezia was observed after starting the treatment and his c-reactive protein levels continued to decline (from 3.2 to 0.16 ). The patient's 
condition gradually stabilized and was subsequently stepped down from the ICU to the ward and discharged 2 weeks later. He received a total of 2 months of Posaconazole therapy. One month post treatment, a follow up colonoscopy did not reveal any lesions (Fig. 3).

\section{Discussion}

One rare cause of lower GI bleeding is GI mucormycosis. Mucormycosis is an opportunistic infection caused by fungi in the order of Mucorales [9]. The low virulence of these organisms makes disease rare among immunocompetent individuals [10]. However, prognosis among infected individuals is extremely poor, and mortality has been found to be associated with the site of infection [9]. Most infected patients have underlying factors of compromised immunity, such as immunosuppression because of organ transplantation, malignancy, persistent neutropenia, poorly controlled diabetes mellitus and systemic corticosteroid therapy $[7,11]$.

Mucormycosis can manifest as different clinical syndromes: rhino-orbito-cerebral, pulmonary, cutaneous, gastrointestinal, disseminated, and uncommon presentations. Among these manifestations, gastrointestinal mucormoycosis is one of the rarest, making up only around 7\% of all cases [10]. Disease of the gastrointestinal tract is usually acquired through ingestion of contaminated food infection of an implanted device. Within the gastrointestinal tract, the stomach is the most common sight of infection, followed by the colon. Mortality of GI mucormyocosis has been reported to be as high as $85 \%$ [12]. Patients often present with nonspecific symptoms such as abdominal pain, GI bleeding, change in

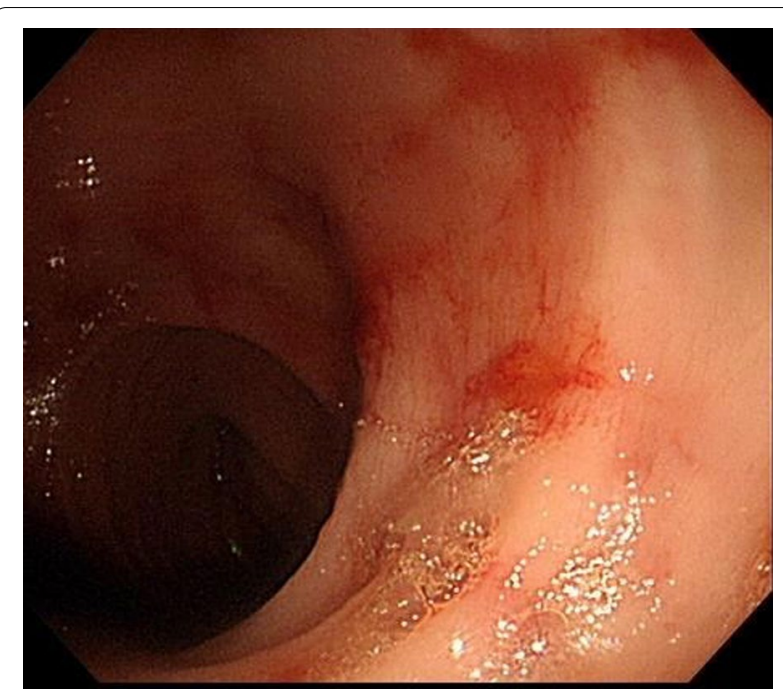

Fig. 3 No lesions were visualized upon follow-up colonoscopy post-treatment bowel habits, and fever [9]. Interestingly, the majority of patients present with abdominal pain in the absence of fever [13]. Nonspecific clinical presentation makes the diagnosis difficult and often delayed, and therefore contributes to the high mortality rate. It is estimated that less than half of the diagnosis of mucormycosis are made antepartum [11].

Definite diagnosis of mucormycosis is made by histopathological evidence of fungal invasion of tissue. Unlike aspergillosis, there is no reliable serological test for mucormycosis. In the case of GI mucormycosis, undergoing invasive endoscopy or even surgery is usually inevitable for diagnosis. A dark appearing ulcer with sharp demarcated edges apparently related to necrosis and thrombosis in adjacent vessels is representative of a typical GI lesion [11]. Endoscopic finding of a small, mushroom-like green mass with a small base attached to the colon wall has also been described in previous studies [14]. Extended lesions can cause perforation, bleeding, and obstruction.

With the widespread availability of endoscopic techniques, early clinical suspicion and diagnosis as well as effective treatment are critical in reducing mortality. However, many patients with severe acute LGIB are unable to tolerate rapid colon preparation for diagnostic colonoscopy, making localization of lesions challenging due to poor visualization. Therefore, in immunocompromised patients such as those with DKA, pharmacological immunosuppression because of organ transplantation, or diseases requiring immunosuppressive medications, it is important to consider the possibility of GI mucormycosis. In the absence of other immediately identifiable etiologies, empiric antifungal treatment should be initiated without delay for clinically suspicious cases of acute severe LGIB in patients who are unable to undergo timely definite diagnosis via colonoscopy.

It is advised that treatment of mucormycosis include urgent surgical resection of infected area whenever feasible parallel to systemic antifungal therapy to prevent disease dissemination $[7,8]$. Liposomal amphotericin B is the recommended first line treatment for mucormycosis. Posaconazole has generally been recommended as maintenance and salvage therapy. However, Song et al. reported a higher survival rate in renal transplant patients with mucormycosis treated with Posaconazole compared to Amphotericin B [15]. The European Confederation of Medical Mycology 2019 guideline proposes the use of Posaconazole as first line treatment with moderate strength, especially in case of renal failure like our patient [16]. Reversal of underlying problems is also fundamental to improve treatment outcome [7, 11]. A study by Gebremariam et al. highlighted the importance of correcting acidemia in patients with ketoacidosis [17]. For 
our patient, proper control of his diabetes mellitus and treatment of alcoholism are of great importance.

\section{Conclusion}

Mucormycosis is a life-threatening fungal infection caused by Mucorales, primarily affecting the immunocompromised hosts. A rare clinical presentation of mucormycosis of the GI tract is hematochezia. Early diagnosis and treatment of mucormycosis infection is critical but can be challenging, especially in the setting of massive hematochezia where adequate bowel preparation for diagnostic colonoscopy cannot be achieved. Therefore, colonic opportunistic infections such as mucormycosis should be a differential diagnosis for massive GI bleeding in immunocompromised patients. In cases with high clinical suspicion, prompt empiric treatment of possible fungal infection is critical.

\begin{abstract}
Abbreviations
LGIB: Lower gastrointestinal bleeding; GI: Gastrointestinal; ED: Emergency department; DKA: Diabetic ketoacidosis; AST: Aspartate transaminase; ALT: Alanine aminotransferase; ICU: Intensive care unit; HBV: Hepatitis B virus; HCV: Hepatitis C virus; HIV: Human immunodeficiency virus; PAS: Periodic acid-Schiff.
\end{abstract}

\section{Acknowledgements}

None declared by the authors.

\section{Authors' contributions}

THC conducted this report and prepared the manuscript. KCF performed the colonoscopy and provided the images. YWL, JHT, CCK, and CCC reviewed the literature. All authors read and approved the final manuscript.

\section{Funding}

None declared by the authors.

\section{Availability of data and materials}

All data generated during this study are included in this published article.

\section{Declarations}

\section{Ethics approval and consent to participate}

The Institutional Review Board of Taipei Medical University Hospital approved this report (IRB No: N202010004).

\section{Consent for publication}

A written consent of personal information for publication was obtained from the patient.

\section{Competing interests}

None declared by the authors.

\footnotetext{
Author details

${ }^{1}$ School of Medicine, College of Medicine, Taipei Medical University, Taipei, Taiwan, ROC. ${ }^{2}$ Division of Gastroenterology, Internal Medicine, Taipei Medical University Hospital, No. 252, Wuxing Street, Taipei, Taiwan, ROC. ${ }^{3}$ Division of Gastroenterology and Hepatology, Department of Internal Medicine, School of Medicine, College of Medicine, Taipei Medical University, Taipei, Taiwan, ROC.
}

Received: 4 January 2021 Accepted: 14 June 2021

Published online: 02 July 2021

\section{References}

1. Strate LL, Gralnek IM. ACG clinical guideline: management of patients with acute lower gastrointestinal bleeding. Am J Gastroenterol. 2016;111(4):459-74.

2. Caballero-Díaz Y, Montesdeoca-Cabrera D, Camacho-Fernández-Pacheco B, Centeno-Haro M, Hernández-Hernández JR. Cytomegalovirus colitis: a cause of severe lower gastrointestinal bleeding. Cir Cir. 2019;87(6):688-91.

3. Siu YP, Tong MK, Kwok YL, Leung KT, Kwan TH, Lam CS, et al. An unusual case of both upper and lower gastrointestinal bleeding in a kidney transplant recipient. Transpl Infect Dis. 2008;10(4):276-9.

4. Bizet J, Cooper CJ, Zuckerman MJ, Torabi A, Mendoza-Ladd A. A bleeding colonic ulcer from invasive Aspergillus infection in an immunocompromised patient: a case report. J Med Case Rep. 2014;8:407.

5. Anand J, Ghazala K, Chong VH. Massive lower gastrointestinal bleeding secondary to colonic mucormycosis. Med J Malays. 2011;66(3):266-7.

6. Shiva Prasad BN, Shenoy A, Nataraj KS. Primary gastrointestinal mucormycosis in an immunocompetent person. J Postgrad Med. 2008;54(3):211-3.

7. Sun M, Hou X, Wang X, Chen G, Zhao Y. Gastrointestinal mucormycosis of the jejunum in an immunocompetent patient: a case report. Medicine (Baltimore). 2017;96(16):e6360.

8. Antony SJ, Parikh MS, Ramirez R, Applebaum B, Friedman G, Do J. Gastrointestinal mucormycosis resulting in a catastrophic outcome in an immunocompetent patient. Infect Dis Rep. 2015;7(3):6031.

9. Serris A, Danion F, Lanternier F. Disease entities in mucormycosis. J Fungi (Basel). 2019;5(1):23.

10. Lo OS-H, Law W-L. Ileocolonic mucormycosis in adult immunocompromised patients: a surgeon's perspective. World J Gastroenterol. 2010;16(9):1165-70.

11. Kumar Debata P, Keshari Panda S, Dash A, Mohanty R, Narayan Mallick B, Tadu D, et al. An unusual presentation of colonic mucormycosis mimicking carcinoma colon - a surgeon's perspective. Int J Surg Case Rep. 2015;10:248-51.

12. Choi HL, Shin YM, Lee KM, Choe KH, Jeon HJ, Sung RH, et al. Bowel infarction due to intestinal mucormycosis in an immunocompetent patient. J Korean Surg Soc. 2012;83(5):325-9.

13. Dioverti MV, Cawcutt KA, Abidi M, Sohail MR, Walker RC, Osmon DR. Gastrointestinal mucormycosis in immunocompromised hosts. Mycoses. 2015;58(12):714-8.

14. Sakorafas GH, Tsolakides G, Grigoriades K, Bakoyiannis CN, Peros G. Colonic mucormycosis: an exceptionally rare cause of massive lower gastrointestinal bleeding. Dig Liver Dis. 2006;38(8):616-7.

15. Song Y, Qiao J, Giovanni G, Liu G, Yang H, Wu J, et al. Mucormycosis in renal transplant recipients: review of 174 reported cases. BMC Infect Dis. 2017;17(1):283.

16. Cornely OA, Alastruey-lzquierdo A, Arenz D, Chen SCA, Dannaoui E, Hochhegger B, et al. Global guideline for the diagnosis and management of mucormycosis: an initiative of the European confederation of medical mycology in cooperation with the mycoses study group education and research consortium. Lancet Infect Dis. 2019;19(12):e405-21.

17. Gebremariam T, Lin L, Liu M, Kontoyiannis DP, French S, Edwards JE Jr, et al. Bicarbonate correction of ketoacidosis alters host-pathogen interactions and alleviates mucormycosis. J Clin Investig. 2016;126(6):2280-94.

\section{Publisher's Note}

Springer Nature remains neutral with regard to jurisdictional claims in published maps and institutional affiliations. 